relationship not only from the perspective of patients, but also from that of therapists. Necessarily the impact that stereotyping and prejudice has on this is heavily emphasised.

This is an easily read book which highlights the importance of empathy gained through the knowledge of others. Although written from the point of view of social workers many of the conclusions drawn are pertinent to anyone working in the field of mental health. Although possibly not 'essential' reading for trainees, I would certainly recommend inclusion of the book in any hospital library. Having read it it made me rethink some of my assumptions and attitudes about the families with whom I work in inner London.

Frankie Connell Senior Registrar in Psychiatry, Tower Hamlets Healthcare NHS Trust, Department of Adult Psychiatry, 3rd Floor Out-Patient Building, The Royal London Hospital, Whitechapel EIB IBB

\section{Cognitive Vulnerability to Depression}

By Rick E. Ingram, Jeanne Miranda \& Zindel V. Segal. New York: Guilford. 1998. 330pp. $£ 26.50$ (hb). ISBN I-57230-304-2

The authors overview existing theories and research addressing cognitive vulnerabilities to depression. Models include Bowlby's development of Adler's hypothesis that anomalies in early attachment (especially uncaring and/or overprotective parents) generate internal working models or cognitive 'schema' that negatively shape processing and interpretation of interpersonal interactions, so inducing and/or maintaining depression. To most clinical psychiatrists, schema models are intuitively appealing, both seemingly confirmed by many patients' reports of their core beliefs as well as allowing common sense therapeutic application. As a consequence, many psychologists and psychiatrists run the theory up the clinical flag pole every day of their professional lives - and despite increasing questioning about the efficacy of cognitivebehavioural therapy (King, 1998).

There is, however, a problem. The theory, not for the first time in the history of psychiatry, resists empirical confirmation. If, as many cognitive therapists have claimed, negative schema are latent constructs intrinsic to those who develop depression and activated by key life events (particularly ones that mirror early adverse events), certain consequences should follow. Some can be noted.

First, prospective studies of those with or without negative cognitive schema should predict onset of depression in the former group when mirroring life event stressors are experienced - a specificity model. Such studies do not appear to have been conducted.

Second, patients with depression in remission should, when 'mood-primed', differ from subjects who are not depressed by the evidence of dysfunctional cognitive patterns. While generally confirmed, such findings do not establish the existence of cognitive schema - as such patterns could equally be a consequence of the state mood disturbance. Third, any such mood-priming should induce consistent schema, an issue apparently not pursued by researchers.

Fourth, returning to the Bowlby hypothesis, if certain parenting behaviours dispose to depression, recall of those behaviours might be expected to identify cognitive vulnerabilities, and the authors note an interesting priming strategy (use of the Parental Bonding Instrument) offering some preliminary support.

Most importantly, patients with depression should, when euthymic, be more likely than subjects who have never suffered from depression to show evidence of ongoing cognitive vulnerabilities. The authors consider the now very large bank of such studies which, almost without exception, fail to reveal such differences. This could reflect over-reliance on two measures which may or may not measure core beliefs and schemas - the Dysfunctional Attitude Scale and the Automatic Thoughts Questionnaire. If not reflecting methodological limitations, and such schema are only evident when an individual is depressed, it is hard to argue for their status as vulnerability factors. The rule of parsimony might then argue for 'schema' as more reflecting state nuances of a depressed mood, a possibility conceded by the authors but rather unconvincingly rejected. Thus, they dismiss a significant challenge to the cognitive Zeitgeist with the ex cathedra statement that there exists "compelling theory and research suggesting that there are important cognitive factors at work in the onset and maintenance of depression" (p. 66). This trifecta of faith, hope and charity is akin to arguing that the Emperor cannot be regarded as naked as he has a large wardrobe at home. Thus, cognitive schema currently appear to have a 'ghost in the machine' status. Schemas, formulated as being 'dormant' or 'latent', thus occupy a position which allows a range of explanations for their 'now you see them, now you don't' status, and which risks being all explanatory. Is it not time for definitive proof of their status or conceptual repositioning - at least as vulnerability factors to depression? Perhaps they have greater relevance to the anxiety and personality disorders rather than to the depressive disorders. If not, why not?

The authors assume that their readers have no knowledge base - at least about depression, cognitive schema, model-testing paradigms or the applied studies. Therefore, this is an excellent reference for students seeking such a primer and a review of the field, but somewhat frustrating to those who have followed the field and who will be impatient for the authors to cut to the chase. The authors impress as 'true believers'; somewhat mystified by the lack of confirmatory research. Rightly so. While this book seeks to inform, its careful preparation raises more questions than answers. That is a noble outcome for an academic product, and worthy of being applauded.

King, R. (1998) Evidence-based practice: where is the evidence? The case of cognitive behaviour therapy and depression. Australian Psychologist, 33, 83-88.

Gordon Parker Professor of Psychiatry, University of New South Wales, Prince of Wales Hospital, Randwick 203I, Sydney, Australia

\section{Panic Disorder}

By D. J. Nutt, J.C. Ballenger \& J.-P. Lépine. London: Martin Dunitz. 1998. 237 pp. Ł49.85. ISBN I-85317-5I8-8

Around half of this book (116 pages) consists of chapters outlining neurobiological theories and drug treatment. The remainder outlines psychological theories and treatments of panic disorder.

The neurobiological perspective is comprehensive. Data are presented from recent radioactive ligand single photon emission computed tomography (SPECT) studies which suggest that alterations at the 